\title{
Reconstruction of Urethra Totally Destroyed in Labour
}

\author{
REGINALD H. J. HAMLIN,* o.B.E., M.A., M.B., F.R.C.o.g. ; E. CATHERINE NICHOLSON,* M.B., B.S., D.oBST.R.C.o.G.
}

\begin{abstract}
Summary : Of 50 women who underwent a reconstruc$\checkmark$ tion procedure for a totally destroyed urethra, caused by obstructed labour, 35 were clinically cured, eight had serious stress incontinence, and six developed urethral stricture; one operation failed completely, but a repeat reconstruction was completely successful. The procedure consists of repair of the bladder fistula, followed by the construction of a new urethra; the latter is then reinforced by one gracilis muscle detached from its insertion and brought through a tunnel in the fascia of the upper thigh and the labium to be attached to the anterior lip of the cervix. Hence this method combines procedures originally suggested for other purposes by IngelmanSundberg and Martius.
\end{abstract}

\section{Introduction}

"The latest fistula to reach me has no urethra at all, and only a very thin fibrous membrane where it formerly was. If one was able to make a new urethra here, how would one make it competent?" (Extract from an East African surgeon's letter to us, October 1966.)

As operative problems fistulas of the birth canal arising from obstructed labour vary from the simplest of injuries easy to repair to those like the one referred to in the quotation above, complicated and formidable. Six main varieties occur: (1) simple vesicovaginal fistula, (2) simple rectovaginal fistula, (3) simple urethrovaginal fistula, (4) difficult high rectovaginal fistula, (5) vesicouterine fistula, and (6) difficult urinary fistula. The last is a complex of several grave injuries occurring together -namely, (a) total destruction of the urethra (all walls) (Figs. 1 and 2), the remaining tissue being merely fibrous connective tissue and squamous epithelium which has grown over and become adherent to the periosteum on the back of the pubic bones; $(b)$ an extensive sloughing of the bladder neck and trigone sometimes so large as to cause one or both ureteric
FIG. 1

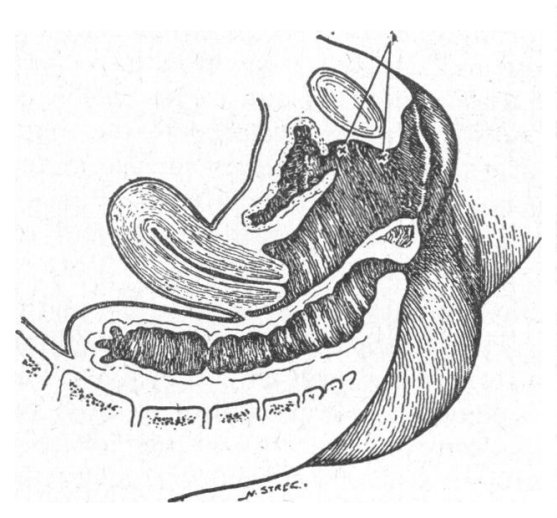

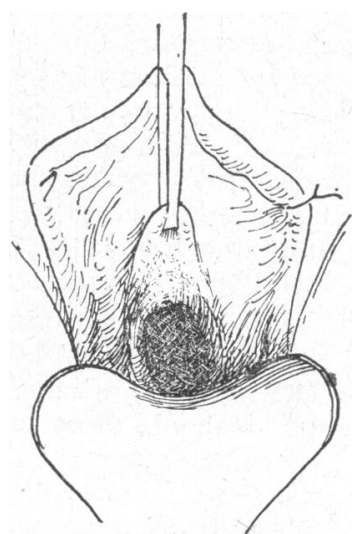

FIG. 2
FIG. 1.-Total destruction of the urethra: diagrammatic representation of the anatomical relationsnips. (From Atlas of Mahfouz's Obstetric and Gynaecological Museum, p. 622. Sherratt, Altrincham.) Fig. 2.-Total destruction of the urethra: only thin, shiny skin covers the area where the urethra previously was. (Redrawn from a photograph.)

* Consultant Obstetrician and Gynaecologist, the Princess Tsahai Memorial Hospital, Addis Ababa. orifices to open directly into the vagina; and (c) fibrosis to an incredible degree which (1) narrows the vagina to the diameter of one fingerbreadth, and (2) binds the remains of the bladder high up to the descending pubic rami and to the pubic symphysis. In a word, no part of the patient's lower urinary tract has escaped some degree of damage.

This is the fistula which daunts the hearts of most observers who see it for the first time. Their immediate reaction is to regard it as incapable of repair. Yet it can be repaired with a complete or at least a satisfactory restoration of function. The purpose of the present paper is to describe its operative management.

But first let us summarize the methods we use in repairing the other five varieties of fistulas-the simpler challenges which everywhere make up the vast majority of obstetric vaginal fistulae.

The following standard textbook procedures have been found suitable and satisfactory for 950 out of the 1,000 fistulas which we have repaired in Addis Ababa: (a) reflection of the vaginal mucosa to expose the fistula, $(b)$ free mobilization of the tissue surrounding the fistula, $(c)$ suturing the fistula with catgut to close it and invert it, (d) labial fat-grafting by Martius's method for all urinary fistulas, and $(e)$ closing the vaginal mucous membrane (everting the edges) with monofilament nylon sutures.

For the remaining $5 \%$ the difficult urinary fistulas (type 6), complicated as they are by an entire loss of the urethra and a complete or almost complete ablation of the vagina due to scarring, another technique of repair is called for. Since the overall incidence of these injuries is in the region of 5 to $6 \%$, anyone repairing fistulas in Africa, in India, in New Guinea, and in other areas throughout the world where obstetric fistulas still commonly occur will encounter them sooner or later. Eight to 10 come to us every year.

\section{Treatment of the Difficult Urinary Fistula (Type 6)}

Professor Naguib Mahfouz (1957) says: “The most troublesome type of all is the case in which the whole urethra has sloughed away." After referring to techniques by Farrar, Gray Ward, etc., he says: "I have done these operations several times but I seldom effected a cure." Chassar Moir (1967) writes: "Obstetrical injuries of a major variety may cause the entire urethra to slough away, even to the extent of leaving little or no tissue to cover the posterior surface of the symphysis pubis. In such cases it is seldom that any worthwhile function can be restored, even by extensive plastic procedures."

The injuries arise (Mahfouz, 1949) in the first stage of labour while the cervix still covers the head of the child, the bladder being distended for one or more days and raised high up behind the symphysis pubis. The urethra, the bladder, the cervix, and especially the area of the bladder near the ureteric orifices, and even the lower ends of the ureters, are all subjected to a prolonged pressure leading to pressure necrosis. Whenever the patient survives so grave an ordeal of obstructed labour, unassisted and unrelieved for several days, healing is followed by scarring, which fixes the remains of these tissues to the back of the symphysis pubis and to the pubic rami. 
The gynaecologist bold enough to attempt the classical flapsplitting operation for a case like this soon discovers that he is operating in an area as confined and almost as inaccessible as the inside of the toe of a leather shoe. He will find himself freeing the bladder of scar and the lateral fixation of its torn edges by touch only. The operator's fingers ache and he is tempted to give up and to transplant the ureters into the bowel, a procedure which we believe should never, or almost never, be resorted to in obstetric fistula surgery. Or he may with fortitude persist, knowing that after he has mobilized and repaired the bladder fistula he will still have to find tissues out of which to construct a urethra that will not necrose but will remain vital and functional. Within the vagina nothing exists for this purpose except, almost quite literally, skin and bone.

\section{Management}

What can be done for injuries so formidable? Our plan of management, developed in a hard experience with 50 challenges of this degree, follows seven steps.

(1) Lithotomy position with a steep Trendelenburg tilt.

(2) A posterolateral incision to open adequately the stenosed vagina.

(3) Incisions in the vaginal wall along the dotted lines in Fig. 3. These incisions demarcate the new urethra that is to be made, and extend around the margins of the large bladder fistula (which is always present where total urethral destruction has occurred). The vaginal wall lateral to the incisions is reflected and the bladder completely released from all scar tissue, especially the anterolateral scar, usually very dense, fixing the bladder to the pubic bones.

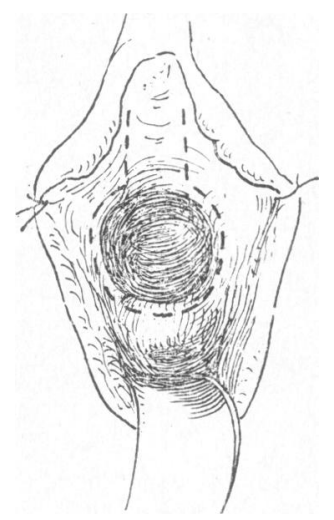

FIG. 3.-Position of the

incisions. (The ureteric

orifices and the cervical

canal are shown for clearness.)

(4) Repair of the bladder defect.

(5) Construction of a new urethra.

(6) Reinforcement of the new urethra by using the gracilis muscle and also, for many cases, the labial fat graft of Martius.

(7) Covering the anterior wall of the vagina and the grafted tissues with mucosa or skin.

The first three steps require no further comment, but steps 4 to 7 do.

\section{Step 4. Repair of Bladder Fistula}

When the bladder has been mobilized the bladder defect is closed with two tiers of interrupted Lembert sutures of No. 00 extra chromic catgut (inverting the approximated edges) over a No. 14 Foley or polyethylene catheter. At the same time we roll the ureteric orifices inside the bladder when they lie on or near the fistula edge.

${ }^{1}$ Irwin-Moore's strong aneurysm is used at the two lateral angles.
Because the bladder fistula is generally large it is often necessary to close it up and down (horizontal suture line), making the needle ${ }^{1}$ penetrate the periosteum or the fascia covering the periosteum on the back of the pubic ramus on each side. This ensures firm anchoring at the lateral angles.

\section{Step 5. Construction of New Urethra}

The inner, or " mucosal," lining of the urethra is made by forming a frail tube from the skin and fibrous connective tissue covering the pubic bones and the inferior border of the symphysis pubis.

Two vertical incisions about $\frac{3}{4}$ in. $(2 \mathrm{~cm}$.) apart in the region of the absent vestibule and along the anterior wall of the lower vagina are made in the skin almost down to the bone, and two skin flaps, left and right, are reflected medially until their free edges can be brought together without tension in the midline beneath the catheter. The approximated skin edges are inverted with two rows of interrupted No. 00 plain catgut, and these sutures convert the two skin flaps into a tube.

The danger-point junction of the new "urethra" with the repaired bladder is reinforced with three or more catgut sutures which draw together folds of bladder muscle over the junction, to lessen the risk of stress incontinence and to invert the junction.

\section{Step 6. Reinforcement of New Urethra}

Such a fragile neourethra, standing unsupported, would almost certainly necrose, and even if it survived would not restore any worthwhile degree of function.

It was the need to find a muscular vital support on which to rebuild urethras totally destroyed by obstructed labour that led us, some seven years ago, to see whether the gracilis muscle, carried as a pedicle graft through a subcutaneous tunnel into th: lower (anterior) vagina, might solve our problem. Looking up the anatomy, we read in Professor R. J. Last's (1954) textbook: "The gracilis muscle does not appear to be an important muscle and the reason for its existence is not very clear. Older anatomists gave the pair of muscles the whimsical name of custodes virginitatis."

The muscle's surface-marking is a straight line running from the pubic spine to the inferior border of the patella. The line is drawn on the skin of the patient's right thigh with "Bonney's blue," the legs being held in the lithotomy position.

An incision about 8 to 10 in. (20 to $25 \mathrm{~cm}$.) long is made on the surface-marking in the middle third of the thigh and the tendon of insertion sought and exposed. No bleeding occurs when the avascular cylindrical tendon is cut. The muscle is freed by light dissection until it remains attached to the thigh only near its origin and at a point on its deeper surface in the upper third, where the nerve and blood supply enter (Fig. 4). It is important that the nerve to the gracilis muscle and its blood vessels are carefully identified and preserved.

Now a tunnel from thigh to introitus is made deep to the skin and superficial fascia of the upper thigh and the labium. The handle of a large scalpel serves well as a director. The tunnel should cross the ischiopubic ramus almost as high up as the level at which the destroyed urethral meatus formerly emerged. The gracilis tendon, after being guided through the sublabial tunnel and under the symphysis pubis into the vagina, is fixed by catgut sutures to the anterior lip of the cervix. In this way the long lower half of the muscle's belly is brought into position under the neourethra as a scaffolding extending from the cervix above to the site of the new urethral meatus below.

Because it is powerfully contractile the gracilis muscle will retract from its vaginal position into the leg unless strong 
catgut sutures (No. 1 chromic) are placed to anchor it firmly to $(a)$ the lateral vaginal fascia, $(b)$ the anterior lip of the cervix, and $(c)$ the fibrous connective tissue covering the periosteum on the medial borders of the ischiopubic rami and the periosteum on the lower anterior surface of the symphysis pubis (Fig. 6).

\section{Step 7}

The final step is to cover the muscle and raw areas with vaginal mucosa. Monofilament nylon sutures are used to unite the mucosal edges in eversion. But very often, as a result of the vaginal stenosis which complicates these cases, all or most of the vaginal mucosa has been destroyed and a new "mucosa" has to be fashioned from pedicle grafts of labial skin swung into position, as high up as possible along the anterior vaginal wall so that the gracilis muscle will be covered over.

In most cases we interpose a secondary reinforcement, “ making security doubly sure," by placing a Martius fat graft between the gracilis muscle and the covering skin or mucosa. Surprisingly the vaginal volume is not much reduced by the introduction of two separate pedicle grafts along the anterior vaginal wall, provided of course that a narrowed vagina-that is, narrowed by the birth damage-has been properly enlarged earlier in the operation.

In a favourable case the operation is completed in two hours, but three hours may be required, especially if a rectovaginal fistula and a complete perineal laceration have to be dealt with as well. Indeed, difficult high or low rectovaginal fistulas and third-degree tears are not rare accompaniments of the difficult urinary fistula.

\section{Comments}

No difficulties arise in transplanting the gracilis muscle. The true problems to be overcome are those created in the vagina by the original extensive birth trauma. Time and patience are needed in opening up the stenosed vagina, in dividing dense scar, and in freely mobilizing and repairing (without tension on the suture line) a bladder whose walls have lost up to onethird or even one-half of their total area. These things must be done without damaging the ureters hidden under scar tissue as tough as cartilage. Occasionally one ureter is found opening outside the bladder and requires reimplantation.
In contrast, making a urethral tube from skin and reinforcing the new urinary channel with the gracilis muscle are simple procedures, and the knowledge that he carries the strong and sure arrow of this technique in his quiver arms the fistula surgeon with a most confident feeling when he is confronted with even the ugliest of challenges.

Bleeding is not troublesome and shock seldom develops. One pint $(570 \mathrm{ml}$.) of compatible blood should, however, be held in readiness, and we often use this support. Low spinal analgesia is always used and is found generally satisfactory. At times supplementary general anaesthesia may be needed.

\section{Results}

We have been pleased with the immediate results. There have been no deaths and only one complete failure of the operation in the series of 50 urethral reconstructions. In this failure the blood and nerve supply to the gracilis muscle were damaged as it was being dissected out.

Relative failure occasionally occurs in the form of pin-point or larger residual urethrovaginal fistulas, which can be easily closed at a second (minor) operation, or they may close spontaneously.

Stress incontinence is met with in $16 \%$ of cases ; a complication which may diminish in time or require a stress incontinence operation. Urethral strictures have brought some patients back to hospital months after the operation. These should all be preventable if we could only afford to keep our patients under careful observation for perhaps six months postoperatively for the regular passage of a urethral sound or dilator.

In summary, the results of these 50 operations are:

(a) One complete failure which was converted to a complete success by a second gracilis operation, using the gracilis muscle of the other leg, six weeks later.

(b) Eight cases of serious stress incontinence (16\%). Four of these patients regained satisfactory continence over a period of some months and four required a stress incontinence operation which restored complete control in two cases and fair control in the other two.

(c) Six patients $(12 \%)$ returned to hospital six months after the operation with a urethral stricture. Threc of these strictures were successfully treated with the passage of a sound or dilator. The other three required operations on the stricture.

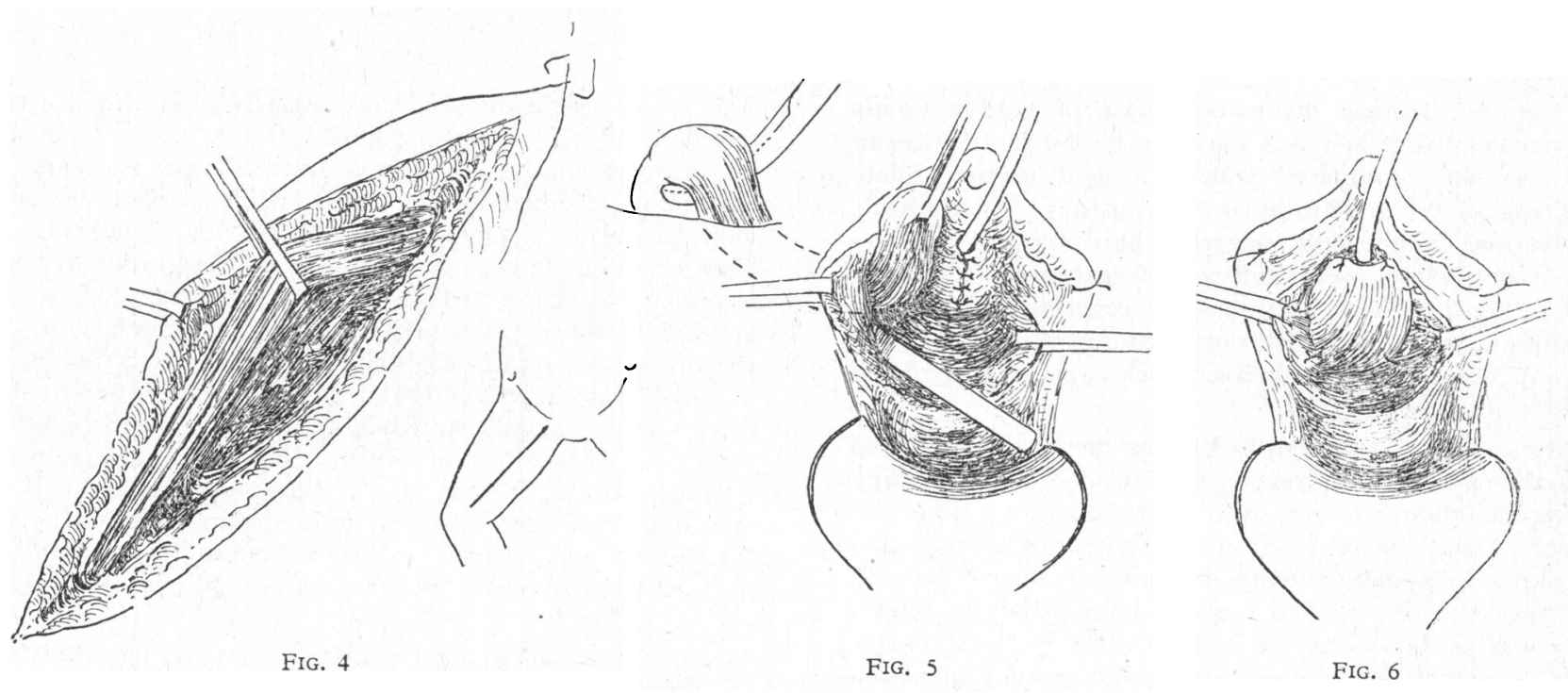

Frg. 4.- The gracilis muscle is exposed and lifted from its bed. Fig. 5.-A " urethra" has becn formed over an indwelling cathcter. A subcutaneous tunnel from thigh to introitus has been made (indicated by the handle of a scalpel), and through this the gracilis muscle is drawn. (Redrawn from a photograph.) FIG. 6. - The gracilis muscle is sutured over the whole length of the new urethra. (Redrawn from a photograph.) 
(d) The remaining 35 patients (70\%)-that is, those who did not suffer either of the complications-went home four to six weeks after the operation clinically cured-that is, completely continent (most patients) or suffering from only a very slight or occasional incontinence of which they made no complaint (a few patients).

\section{Pioneering Work}

We are indebted to Professor Ingelman-Sundberg, of Stockholm, and to Professor Martius, of Göttingen. Our technique borrows essential features from their pioneering work and we are very glad indeed to have this opportunity of recording our indebtedness to them both.

For nine years we have been using Martius's labial fatgrafting procedure in the repair of urinary fistulas not complicated by a total loss of the urethra. Seven years ago we read in Chassar Moir's (1956) Joseph Price oration that IngelmanSundberg had designed a remarkable operation in which the gracilis muscle was used as a pedicle graft directed through the obturator foramen into the upper vagina as a means of closing radium-produced bladder fistula.

Moir's report of Ingelman-Sundberg's brilliant work suggested to us that the solution to our problem-the totally destroyed urethra-might at last be found if we could combine the virtues of Professor Ingelman-Sundberg's muscle graft with those of Professor Martius's route. Two questions answerable only by a trial operation arose: (1) was the average gracilis muscle long enough to be directed into the vagina via Martius's indirect channel? and (2) if so would it provide the active muscular floor and walls of a reconstructed urethra?

The trial operation, undertaken in 1961, was happily a success. It gave favourable answers to both questions ; and many opera- tions of the same pattern since then have confirmed that the procedure does enable destroyed urethras to be soundly reconstructed with restoration of continence in the majority of cases.

Chassar Moir (1965), after seeing the operation in Addis Ababa four years ago, wrote: "The methods used by IngelmanSundberg and by Hamlin and Nicholson are essentially different. In the former the gracilis muscle is brought by a deep route to the upper vagina, where it provides a new blood supply and serves as a plug for the fistula; in the latter the muscle is brought by a superficial route into the lower vagina, where it provides a direct support to the bladder and urethral walls."

Our debt to Professor Ingelman-Sundberg and also to Professor Martius is obvious and great, and to them both we have expressed our gratitude earlier in this paper.

To two other fistula surgeons known throughout the world we are also much indebted-Professor Naguib Mahfouz and Professor Chassar Moir. In frequent letters over many years they have encouraged us and advised us in all our technical problems. Almost everything valuable about fistula surgery has been beautifully described or reported on in their writings. We salute them.

\section{REFERENCES}

Moir, J. Chassar (1967). Vesico-vaginal Fistula, 2nd ed., p. 108. London, Baillière, Tindall and Cassell.

Moir, J. Chassar (1965). Journal of Obstetrics and Gynaecology of India, 15,441 .

Moir, J. Chassar (1956). American fournal of Obstetrics and Gynecology, $71,476$.

Mahfouz, N. (1949). Atlas of Mahfouz's Obstetric and Gynaecological Museum, vol. 2. Altrincham, Sherratt.

Mahfouz, N. (1957). Fournal of Obstetrics and Gynaecology of the British Empire, 64, 23.

Last, R. J. (1954). Anatomy, Regional and Applied. London, Churchill.

\title{
Serum and Urinary Folate in Liver Disease
}

\author{
F. P. RETIEF,* M.D., D.PHIL., M.R.C.P. ; Y. J. HUSKISSON, † B.SC.
}

$S^{u}$ ummary : During the active phase of viral hepatitis urinary folate loss was found to be 8.0 to 48.3 (mean 31.1) $\mu \mathrm{g}$./day, compared with a normal urinary folate excretion of 0.1 to 18.0 (mean 9.5) $\mu \mathrm{g}$./day. In cirrhosis and cardiac failure with congestive hepatomegaly the corresponding values were 25.8 to 55.0 (mean 35.7 ) $\mu$ g./day and 2.5 to 61.6 (mean 26.9) $\mu \mathrm{g}$./day, respectively. Urinary folate loss may be a significant factor in the aetiology of folate deficiency of chronic liver disease, particularly when dietary intake is poor.

After prolonged dialysis in Visking casing urinary folate was almost totally dialysable, but an appreciable fraction of serum folate was not, even after 72 hours. The dialysable (free) folate fraction of serum and urine disappeared maximally during the first six hours' dialysis, and was virtually cleared after 24 hours' dialysis ; clearance curves in normal individuals and in liver disease were comparable. The non-dialysable serum folate fraction was of similar magnitude in all subjects studied, in spite of marked variation in total folate, and probably represented protein-bound folate.
The folate deficiency of liver disease is of multiple aetiology (Herbert, 1965). It is commonly the result of poor dietary intake, particularly in chronic liver disease associated with alcoholism (Herbert et al., 1963), while alcohol per se may increase folate requirement (Sullivan and Herbert, 1964). Haemolysis of chronic liver disease and erythroid hyperplasia secondary to haemorrhage from oesophageal varices causes increased folate demands (Klipstein and Lindenbaum, 1965). In cirrhosis enzymes essential for folate metabolism may be deficient (Carter et al., 1961 ; Merritt et al., 1962). In the adult folate is mainly stored in the liver in quantities of about 5-20 mg. (Chanarin et al., 1966). Hepatic dysfunction affects vitamin storage (Baker et al., 1964), and vitamin $B_{12}$, for instance, is liberated into the blood stream during liver disease (Stevenson and Beard, 1959). Vitamin $\mathrm{B}_{12}$ is effectively retained by plasma binders, resulting in significant urinary loss

* Head of Haematology Division, Department of Internal Medicine, Stellenbosch University Medical Schaol; Senior Consultant, Karl Bremer Hospital, Bellville, C.P., South Africa.

† Research Assistant, Haematology Division, Department of Internal Medicine, Stellenbosch University Medical School, Bellville, C.P., South Africa. 